\title{
Surface active properties of silicate and aluminosilicate surfaces
}

\author{
Dmitry Bondarenko, Iryna Plakhotnikova, Medeia Saliia*, Olga Demina, \\ and Alexander Bondarenko \\ Kharkiv National University of Civil Engineering and Architecture, 61002 Sumska st, 40, Kharkiv, \\ Ukraine
}

\begin{abstract}
Liquid heat-insulation based on hollow microspheres and polymer compounds become more and more popular in the construction industry. This material enjoys indisputable technological advantages easiness of application, low mass, flexibility, good adhesion to different materials. Traditional non-organic heat insulating materials, widely presented on modern construction market, have some disadvantages. Foam concrete and gas silicate have relatively high average density, which enables obtaining items and materials with a fairly low thermal conductivity coefficient. These materials have high saturation coefficient and low values of cold endurance. Inside the mineral-wool plates, which are quite popular on the modern market, the hyperfine fibers transform into dust particles by time, and agglutinative compounds destruct, releasing toxic substances. The aluminosilicate microspheres have a high potential as a commercial product, but their market has just started forming. According to rough calculations, the cost of the product is next lower order to the hollow microspheres, obtained by industrial methods.
\end{abstract}

\section{Introduction}

The requirements for ecological friendliness and durability of the construction materials are increasing rapidly. As a result, effective and ecologically safe materials are getting increasing demand and actuality [1 - 3].

One of these materials can be thin-layered coating based on a mineral binder or a polymeric compound, filled with glass or aluminosilicate hollow microspheres. Functional properties of such materials spark off a lot of questions among the experts. Firstly, it is about the thermal conduction coefficient, which is varying among manufacturers between $0.022-0.057 \mathrm{~V} / \mathrm{mK}[2]$.

The second issue is the principles of heat transfer. For traditional heat-insulating materials, the mechanism is connected with the photons. The function of the materials, filled with hollow microspheres made of glass or aluminosilicate up to $90 \%$ and more, lies in the fact of a threefold mechanism of heat transmission: by the photons inside the material itself; by air convection; and by reflection of the main types of radiation, including heat.

*Corresponding author: medeasalia@gmail.com 
The reflection effect increases by the presence of inner partitions inside the microspheres, which slows down air convection. There is a great amount of light ash and slugger, occurring at the heat power plants, which is currently accumulating around the world. An upcoming trend of this kind of waste utilization is obtaining and application of the hollow microspheres. They are glass-like hollow spherical particles, occurring by combustion of coal from certain deposits in the caldrons of the heat power stations. The value of the microspheres is caused by their low bulk density, low heat conductivity and spherical form of the particles. In average, the concentration of microspheres in fly ash can fluctuate from 0.3 to $3-5 \%$. Significantly, it is relatively easy to extract the hollow microspheres out of the ash. Because of low density, they surface in waterworks of a heat power plant and it is easy to collect them. The aluminosilicate microspheres (ASM) have a high potential as a commercial product, but their market has just started forming. According to rough calculations, the cost of the product is next lower order to the hollow microspheres, obtained by industrial methods. Application of ASM has a positive ecological effect because it enables the utilization of the wastes of production.

\section{Materials and methods of research}

It is known, that effective heat insulating materials are obtained from the expanded perlite (slabs, plasters), the grains of which have septate structure, and this structure allows decreasing heat transferring inside the material by means of convective heat. Microscopic researches have been carried out during the execution of the work; it has been shown that the structure of the glass and aluminosilicate microspheres also belong to a developed septate type.

This work investigates the possibilities of glass and aluminosilicate microspheres application in cement compounds. Exposition in alkaline condition by $\mathrm{pH} 12$ within 28 days has shown that the surfaces of microspheres remain intact, Fig.1-2.

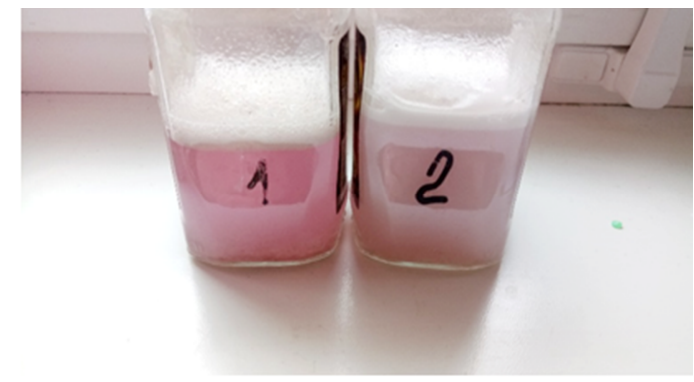

Fig. 1. Exposition of glass and aluminosilicate microspheres in water condition by $\mathrm{pH}=12$.

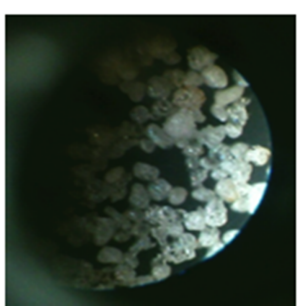

(a)

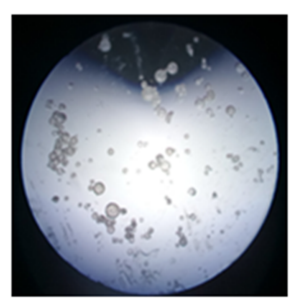

(b)

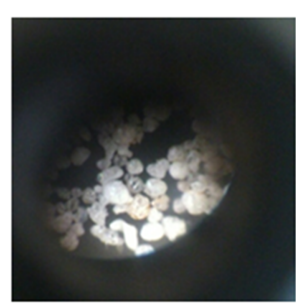

(c)

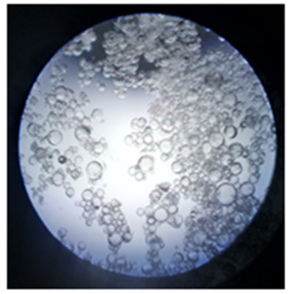

(d)

Fig. 2. The research of surface condition in glass and aliminosilicate microspheres before exposition $(a, b)$, and afterwards (c, d). 
Surface properties of the microspheres have been detected quantitatively and qualitatively by means of the technique, described in works by A.P. Nechyporenko, V.A. Matviienko, V.I. Babushkin, A.A. Plugin and others [4-8]. Changes in the electronic system of the microspheres surface under the influence of various factors bring about a shift of the acid-alkali balance in the respective direction, which express in the change of the dissociation constant, $\mathrm{Kd}$. The surface of a solid substance is a combination of established acids and alkali, which amount and strength can be fixed by means of molecular indicators adsorption and expressed by their $\mathrm{Kd}$, i.e. in $\mathrm{pH}$ scale units.

The research, carried out in this work, namely detection of adsorption centers on the surface of the aluminosilicate microspheres, has shown the following. The concentration of the Bronsted base centers (of negative surface charge) comprises $23.1 \times 105 \mathrm{mg} \mathrm{eq} / \mathrm{g}$. In cement stone, this value comprises $54 \times 105 \mathrm{mg}$ eq/g, that is 2.3 times more. The concentration of the Lewis base centers (of positive surface charge) comprises in aluminosilicate microspheres $4.5 \times 105 \mathrm{mg}$ eq $/ \mathrm{g}$, against $0.7 \times 105 \mathrm{mg} \mathrm{eq} / \mathrm{g}$ in cement stone, which is 6 times more. These balance of active adsorption centers in cement particles and aluminosilicate microspheres allowed strengthening of intergranular space in cement composite by forming silicate gel over their surfaces that has been proven by electronic microscopy researches, Fig. 3.

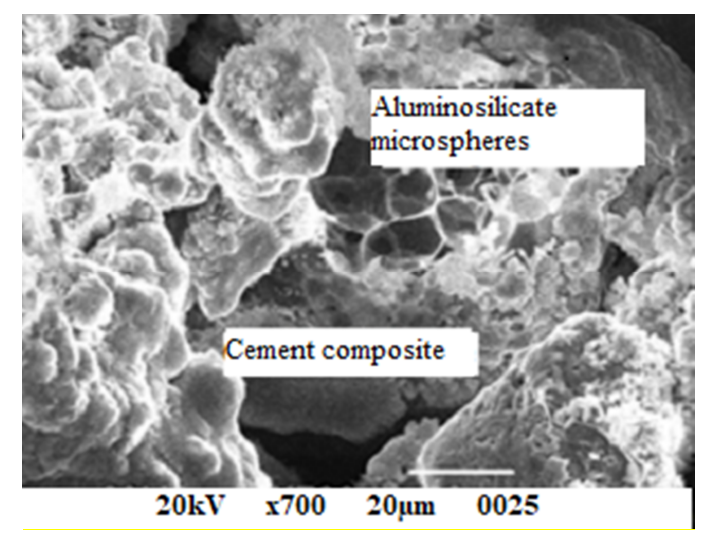

Fig. 3. Electronic microscopy pictures of cement composite with aluminosilicate microspheres on the seventh day.

In order to provide the highest structural strength and to narrow down the intergranular space, it is necessary to create the balance between the particles of the opposite surface charge. It was shown by previous studies that on the silicate base layers of glass microfibers in cement stone, the formation of the crystalline hydrates takes place, and the compounds of positive surface charge are the hydroaluminates and calcium carbonate, Fig. 4.
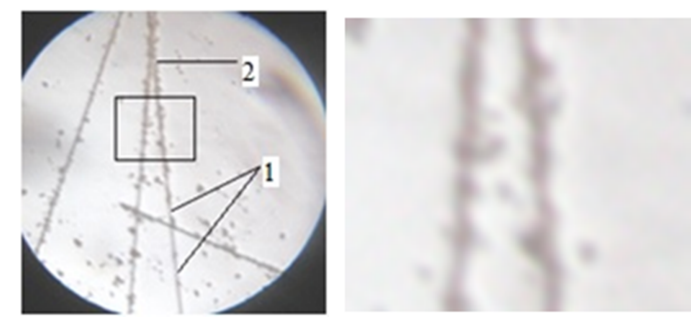

Fig. 4. Formation of the crystalline hydrates with a positive surface charge on silicate surface of a glass microfiber: 1 - a glass microfiber (enlargement 96x), 2 - the crystalline hydrates fouling of a glass microfiber on the 28th day in cement extract. 
Equally spread inside the mass, the process of formation of crystals with positive and negative surface charge, allows obtaining more solid and hard structure, Fig.5.

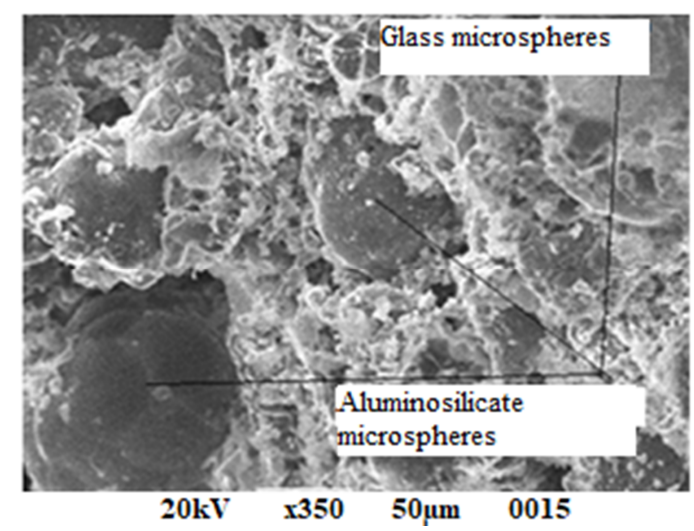

Fig. 5. Electronic microscopy pictures of cement composite, containing silicate and aluminosilicate hollow microspheres on the 28th day.

Electrical surface properties of the components, comprising the system of dry construction mixtures, which we research, have been checked under the impact of an electrostatic field of high voltage by the Razryad device following the technique [8, 10-12]. Fig. 5 shows a device for quantitative evaluation of surface polarity by separation of particles in high voltage field [13].

\section{Conclusions}

In order to provide the highest durability in cement composites, obtaining the balance between positively and negatively charged surfaces of all dispersive particles and crystalline hydrates is necessary. Adding 5-50 $\mu \mathrm{m}$ glass and aluminosilicate microspheres to cement structure shows the positive impact on the formation of the more dense structure as on early stages, as in later terms of solidification.

\section{References}

1. V. Demchenko, V. Sviders'kyi. Continuous development, its condition, and perspectives, International Scientific Symposium SDEV'2018 Materials. Heat insulating building mixtures with modified ash microspheres, 205-208 (2018)

2. The myths of cosmic technologies or marketers fantasies https://www.microspheres.com.ua

3. K.V. Plakhotnikov, O.I. Bondarenko, O.B. Dedeniova. Research journal of building industry, 3 (89), 226-229 (2017)

4. A.P. Netchyporenko, A.I. Kudriashova. Journal of Applied Chemistry, 9 (60) (19571961)

5. V.I. Babushkin, A.A. Plugin, T.A. Kostyuk, V.A. Matviyenko, Research journal of building industry, 5, 85-88 (1998)

6. V.M. Vorobiov, D.R. Kadyrova, G.Sh. Talipov. Kinetics, and catalysis, 1(15), 170-175 (1974)

7. V.A. Matviienko, Electrical activation in the technology of concrete production. $\mathrm{PhD}$ sci. diss. (Makiyivka, 1993) 
8. A.A. Plugin, Research Journal of NTU KhPI, 15 (2003)

9. Saliia M.G. Waterproofing coating on a cement base with increased crack resistance. $\mathrm{PhD}$ sci. diss. (Kharkiv, 2012)

10. A.A. Plugin, Longevity of concrete and reinforced concrete in drown constructions. Colloid chemical foundations. Doctoral dissertation in technical sciences (Kharkiv, 2005)

11. A.N. Plugin, Electrical heterogenous interaction when hardening of cement binders. Doctoral dissertation in chemistry, 282 (Kyiv, 1989)

12. O.I. Demina, A.A. Plugin, E.B. Dedenyova, D.O. Bondarenko, T.A. Kostuk, Functional Materials, 3(24), 415-419 (2017)

13. A.A. Plugin, Journal of the NTU «KhPI», 33 (2004) 